\title{
SÍNTESE DE ANÁlOgo DE BRASSINOESTERóIDE A PARTIR DE VESPERTILINA
}

José L. M. Gárate e Gouvan C. de Magalhães

Departamento de Química - Universidade de Brasília - 70910-900 Brasília - DF

Luiz A. S. Romeiro

Departamento de Química - Universidade de Brasília - 70910-900 Brasília - DF

Departamento de Física e Química - Universidade Católica de Brasília - QS 07 Lote 01 EPCT - Águas Claras - 72022-900 -

Taguatinga - Brasília -DF

Recebido em 25/8/97; aceito em 14/4/98

\begin{abstract}
SYNTHESIS OF A BRASSINOSTEROID ANALOGUE FROM VESPERTILINE. In the last years, several research groups have been working on the synthesis of new steroidal plant hormones called brassinosteroids (BS), which promote plant growth and better crops. Many synthetic targets and applications of these compounds and their analogues have been described in the literature. From Solanum species of the Distrito Federal, we isolated the steroidal alkaloid solasodine, which was then converted into our starting material, vespertiline. By functionalization of rings $A$ and $B$, we have synthesized a new analogue of $B S$, with a $2 \alpha, 3 \alpha$-dihydroxy-6-one structure, typical of the naturally occurring $B S$ castasterone, the immediate biosynthetic precursor of brassinolide.
\end{abstract}

Keywords: vespertiline; brassinosteroids; plant growth-promoting steroids.

\section{INTRODUÇÃO}

A partir de 1970, diversos grupos de trabalhos divulgaram que extratos lipossolúveis de muitas fontes vegetais, continham substâncias que promoviam alongação e divisão celular de plan$\operatorname{tas}^{1-3}$. A descoberta e determinação estrutural de um novo tetrahidróxi-esteróide, isolado do pólen da planta Brassica napus L., denominado brassinolídeo, marcou o início de um novo período de investigações de sistemas hormonais reguladores do crescimento de organismos vegetais ${ }^{4-6}$. A característica estrutural deste novo composto, determinada através de cristalografia de raio- $X$, consiste na presença de um grupo metila no carbono, C-24, de configuração S, uma lactona 6-ceto-7-oxa no anel $\mathrm{B}$ e hidroxilas vicinais, $2 \alpha, 3 \alpha$ no anel $\mathrm{A}$ e nos carbonos, $\mathrm{C}-22$ e C-23, de configuração $\mathrm{R}^{7-8}$.

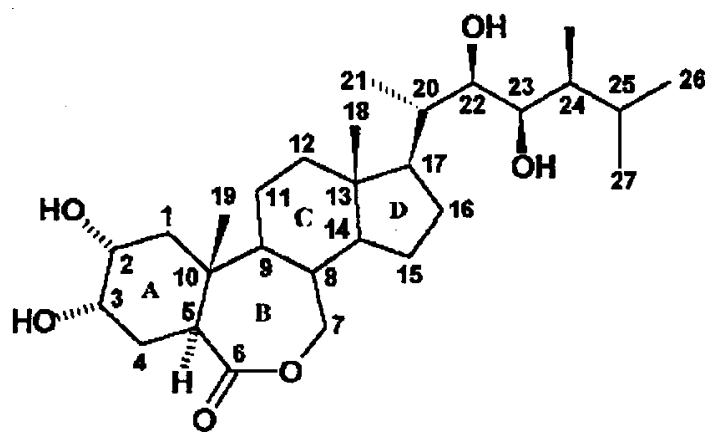

A partir de diversas fontes vegetais foram isolados vários compostos que possuem similaridades estruturais e funcionais ao brassinolídeo. Estes compostos, que receberam o nome genérico de Brassinoesteróides (BS), constituem uma nova classe de fito-hormônios, responsáveis por promover o crescimento de plantas ${ }^{9-13}$. Todos os BS de ocorrência natural são derivados de $5 \alpha$-colestanos e estão inseridos em grupos de esteróides contendo de 27 a 29 átomos de carbono, como fitoesteróides típicos. Os BS estão ainda agrupados, de acordo com os estágios de oxidação do anel B, em 7-oxa-lactônicos, 6-cetônicos, e os não oxidados (Fig. 1) ${ }^{14,15}$.

Entre as principais características apresentadas pelos BS podemos citar a habilidade de atuar com um potente efeito biológico sobre a balança hormonal em concentrações muito baixas ( $\left.5 \times 10^{-4}-5 \times 10^{-3} \mu \mathrm{g} / \mathrm{mL}\right)$, ativar os processos metabólico e de crescimento, aumentar a produtividade total da planta e melhorar sua resistência a condições climáticas adversas. A este conjunto de propriedades deu-se o nome de atividade brassínica.

O interesse pela síntese de BS foi despertado por uma série de fatores, entre os quais citamos a baixa concentração nas fontes naturais $\left(10^{-5}-10^{-12} \%\right)$, tornando difícil e de alto custo o seu isolamento e os efeitos notáveis apresentados sobre os organismos vegetais em concentrações muito baixas ${ }^{16}$

Já é possível encontrar na literatura estudos que estabelecem algumas relações estrutura/atividade para se obter uma alta atividade brassínica ${ }^{17,18}$. Sabe-se que é necessário a existência de dois grupos hidroxila nas posições $22 \mathrm{R}$, 23R da cadeia lateral, bem como a presença de um grupo metila ou etila $24 \mathrm{~S}$ e de um grupo hidroxila $3 \alpha$. Verificou-se ainda que os compostos 7-oxa-lactônicos são mais ativos que os compostos 6cetônicos, bem como os compostos com um grupo lactona 6ceto-7-oxa (lactona normal) são mais ativos que os compostos que possuem a lactona 7-ceto-6-oxa (lactona isomérica). O decréscimo de atividade para os dióis segue a ordem: $2 \alpha, 3 \alpha>$ $2 \alpha, 3 \beta>2 \beta, 3 \alpha>2 \beta, 3 \beta$.

Apesar de importantes, estas características gerais não são rígidas, uma vez que vários exemplos citados na literatura apresentam estruturas que possuem atividade brassínica e não apresentam todas as funções citadas ${ }^{19}$.

$\mathrm{O}$ isolamento do alcalóide esteroidal solasodina, a partir de espécies de Solanum do DF, vem sendo efetuado em nosso laboratório desde 1993, com o objetivo de sua transformação em 16-desidropregnenolona, matéria prima de fármacos esteroidais. Nosso interesse na química de brassinoesteróides surgiu da possibilidade de sintetizar análogos de BS a partir da vespertilina, baseados nas atividades mostradas por alguns análogos de BS para culturas de arroz e feijão. Estes análogos de BS foram sintetizados a partir da diosgenina e derivados pregnanos e androstanos ${ }^{19,20}$. 
<smiles>C=C(C(C)C)[C@H](O)[C@H](O)C(C)C1CCC2C3COC(=O)[C@]4(CCCC12C)C[C@H](O)C(O)CC34C</smiles>

Dolicólido<smiles>CC(C)C[C@H](O)[C@H](O)C(C)C1CCC2C3COC(=O)[C@]4(CCCC12C)C[C@H](O)C(O)CC34C</smiles>

28-Norbrassinólido<smiles>CC(C)[C@H](C)[C@H](O)[C@H](O)C(C)C1CCC2C3CC(=O)[C@H]4C[C@H](O)[C@@H](O)C[C@]4(C)C3CCC21C</smiles>

24-Epicastasterona<smiles>C=C(C(C)C)[C@H](O)[C@H](O)[C@H](C)C1CCC2C3CC[C@H]4C[C@H](O)[C@@H](O)CC4(C)C3CCC21C</smiles>

6-Desoxodiidrodolicosterona<smiles>C/C=C(/C(C)C)[C@@H](O)[C@H](O)C(C)C1CCC2C3COC(=O)[C@]4(CCC[C@]12C)CC(O)C(O)CC34C</smiles>

Homodolicólido<smiles>C=C(C(C)C)[C@H](O)[C@H](O)C(C)C1CCC2C3CC(=O)[C@H]4CC(O)C(O)CC4(C)C3CCC21C</smiles>

Dolicosterona<smiles>CC(C)C[C@H](O)[C@H](O)C(C)C1CCC2C3CC(=O)[C@H]4CC(O)C(O)CC4(C)C3CCC21C</smiles>

Brassinona<smiles>C/C=C(\C(C)C)[C@@H](O)[C@H](O)[C@H](C)[C@H]1CCC2C3CC[C@H]4C[C@H](O)[C@@H](O)C[C@]4(C)C3CC[C@]21C</smiles>

6-Desoxodiidrohomodolicosterona<smiles>CC(C(O)[C@@H](O)[C@@H](C)C(C)C)C1CCC2C3COC(=O)[C@]4(CCCC12C)CC(O)[C@H](O)CC34C</smiles>

24-Epibrassinólido<smiles>C/C=C(\C(C)C)[C@@H](O)[C@H](O)[C@H](C)[C@H]1CCC2C3CC(=O)[C@H]4CC(O)[C@@H](O)CC4(C)C3CCC21C</smiles>

Homodolicosterona<smiles>CC(C)[C@H](C)[C@H](O)[C@@H](O)[C@H](C)[C@H]1CCC2C3CC[C@H]4C[C@H](O)[C@@H](O)C[C@]4(C)C3CC[C@]21C</smiles>

6-Desoxodiidrocastasterona<smiles>CC(C)[C@H](C)[C@H](O)[C@H](O)[C@H](C)[C@H]1CCC2C3CC[C@H]4C[C@H](O)[C@@H](O)C[C@]4(C)C3CC[C@]21C</smiles>

3-Epi-6-desoxodiidrocastasterona

Figura 1. Estágios de oxidação no anel $B$ de brassinoesteróides.

\section{RESULTADOS E DISCUSSÃO}

Neste trabalho, apresentamos os resultados obtidos a partir das modificações nos anéis A e B da estrutura da vespertilina, para obtenção de um análogo de BS, com as características funcionais, $2 \alpha, 3 \alpha$-di-hidróxi-6-ceto, típica da castasterona, brassinoesteróide de ocorrência natural.

Iniciamos nosso trabalho sintetizando, a partir do alcalóide esteroidal solasodina $^{21}$, a lactona 22,16 do ácido $3 \beta, 16 \beta$-dihidróxi-5-bisnorcolênico (vespertilina), utilizando as etapas sintéticas publicadas por Sato e colaboradores ${ }^{22,23}$. Os rendimentos e a pureza dos intermediários e do produto final estão de acordo com os dados da literatura utilizada.

A primeira etapa na preparação do análogo de BS consistiu em transformar o grupo $3 \beta-\mathrm{OH}$ em um bom grupo abandonador por tosilação.

Seguindo a rota planejada (esquema 3), preparamos o derivado 3,5-ciclo-6-hidróxi-vespertilina (V2), por solvólise do tosilato de vespertilina. O mecanismo do rearranjo à 3,5-ciclo-esteróides a partir solvólise de tosilatos está demonstrado no esquema $1^{24}$.
Introduzimos algumas modificações no procedimento de elaboração desta reação descrita no trabalho de Steele e Mossetig, ${ }^{25} \mathrm{com}$ as quais diminuímos o tempo elaboração de 24 a 48 horas para 1 hora. A oxidação de V2, foi efetuada com o reagente de Jones, onde obtivemos V3 com rendimento de 98\% muito superior ao da literatura

A etapa seguinte consistiu na clivagem do anel ciclopropano e formação da dupla 2,3 no anel A. Para esta reação utilizamos o procedimento descrito por Mori e colaboradores ${ }^{26}$.

Uma proposta mecanística da clivagem do anel ciclopropano e formação da dupla $\Delta^{2}$ pode ser representada como mostrado no esquema 2.

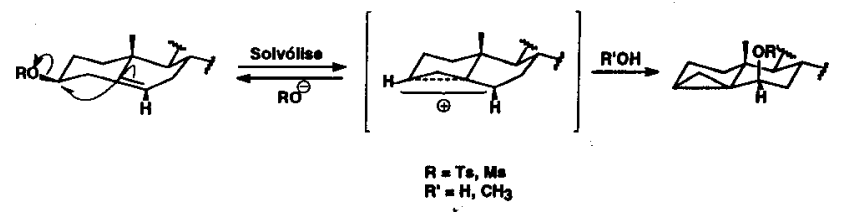

Esquema 1. Mecanismo da formação de 3,5-ciclo-esteróides por solvólise 


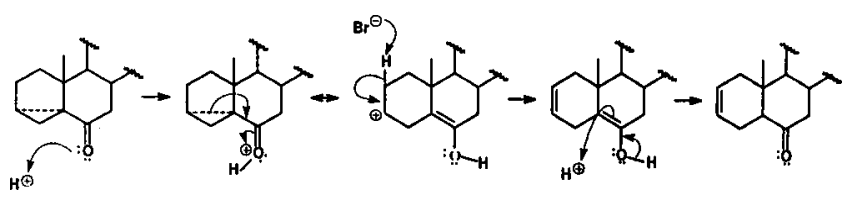

Esquema 2. Proposta de fissão do anel ciclopropano e formação de $\Delta^{2}$

A oxidação da dupla $\Delta^{2}$, visando a obtenção do análogo de BS $2 \alpha, 3 \alpha$-di-hidróxi-6-ceto-vespertilina (V5), foi realizada com tetróxido de ósmio.

Os resultados da rota sintética desenvolvida para obtenção do análogo de BS a partir de vespertilina estão resumidos no esquema 3 .
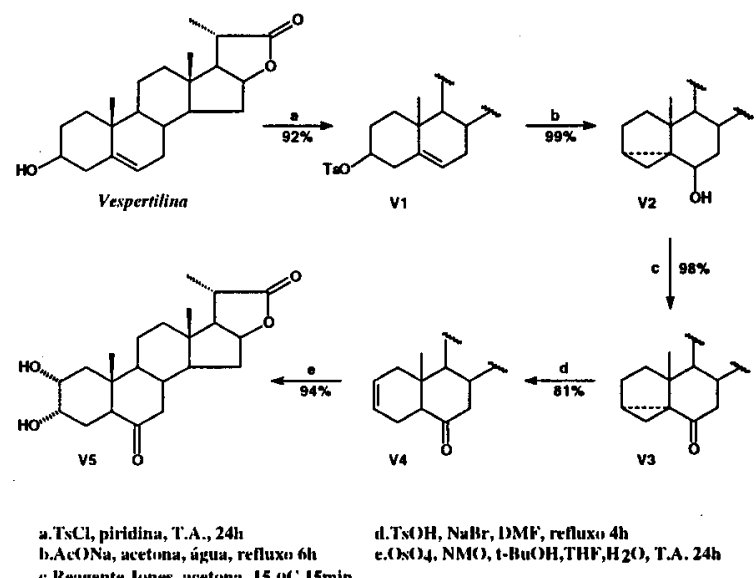

I.Ts()H, Na Br, 1)Mr, relluxu, 4h

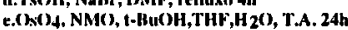

Esquema 3. Resultados da rota sintética desenvolvida para obtenção de análogo de BS a partir de vespertilina

\section{PARTE EXPERIMENTAL}

Os reagentes e solventes P.A. (Merck, Aldrich, Fluka, Vetec) foram utilizados sem purificação prévia, exceto quando a reação exigia condições anidras.

O controle das reações foi realizado através de cromatografia em placa de camada delgada (CCD), empregando silicagel $60 \mathrm{~F} 254$ suportada em alumínio (Merck), utilizando como reveladores: a) vapores de iodo, b) solução $5 \%$ de ácido fosfomolíbdico em etanol e c) solução de vanilina em ácido sulfúrico $\left(\mathrm{d}=1,25 \mathrm{~g} \mathrm{~mL}^{-1}\right) /$ etanol.

Os espectros no infravermelho (IV) foram registrados no espectrômetro Nicolet 5ZDX-FT e no Bomem Hartmann \& Brawn MB-Series.

Os pontos de fusão foram determinados no bloco de Köfler e estão registrados sem correção.

O material de partida, vespertilina, foi obtido por degradação do alcalóide esteroidal solasodina, isolado de frutos de espécies de Solanum coletadas no Distrito Federal. A vespertilina apresentou p.f. $225-226^{\circ} \mathrm{C}$, alta pureza por CCD e dados de Infravermelho e RMN ${ }^{1} \mathrm{H}$ e ${ }^{13} \mathrm{C}$ idênticos aos descritos na literatura.

IV $(\mathrm{KBr}) \mathrm{n}_{\text {máx }} \mathrm{cm}^{-1}: 3440\left(\mathrm{v}_{\mathrm{OH}}\right), 2947-2845\left(\mathrm{v}_{\mathrm{CH}^{-}} \mathrm{sp}^{3}\right), 1668$ $\left(v_{\mathrm{C}=\mathrm{C}}\right), 1770\left(v_{\mathrm{CO}}\right.$-éster $), 1452-1343\left(\delta_{\mathrm{CH} 2}+{ }_{\mathrm{CH} 3}\right)$

$\mathrm{RMN}{ }^{1} \mathrm{H}\left(\mathrm{CDCl}_{3} 200 \mathrm{MHz}\right) \delta: 5,35(\mathrm{~m}, \mathrm{H}-6), 4,93(\mathrm{~m}, \mathrm{H}-16)$ 3,50 (m, H-3), 1,30 (d, 3H-21), 1,02 (s, 3H-19) 0,75 (s, 3H-18).

$\mathrm{RMN}{ }^{13} \mathrm{C}\left(\mathrm{CDCl}_{3} 50 \mathrm{MHz}\right) \delta: 181,4(\mathrm{C}-22), 140,8(\mathrm{C}-5)$, 120,8 (CH-6), 82,7 (CH-16), 71,4 (CH-3), 58,7 (CH-17), 54,6 (CH-14), 49,9 (CH-9), 42,0 ( $\left.\mathrm{CH}_{2}-4\right), 41,3(\mathrm{C}-13), 38,0\left(\mathrm{CH}_{2}-\right.$ 12), 37,1 ( $\left.\mathrm{CH}_{2}-1\right), 36,4(\mathrm{C}-10), 35,9(\mathrm{CH}-20) 32,9\left(\mathrm{CH}_{2}-15\right)$, $31,7\left(\mathrm{CH}_{2}-7\right), 31,3\left(\mathrm{CH}_{2}-2\right), 31,1(\mathrm{CH}-8) 20,2\left(\mathrm{CH}_{2}-11\right), 19,2$ $\left(\mathrm{CH}_{3}-19\right), 16,4\left(\mathrm{CH}_{3}-18\right), 13,6\left(\mathrm{CH}_{3}-21\right)$.

\section{Tosilato de vespertilina (V1)}

Em um balão $(150 \mathrm{~mL})$, foram adicionados vespertilina $(2,0$ $\mathrm{g}, 5,80 \mathrm{mmol})$, piridina $(60 \mathrm{~mL}$, tratada com $\mathrm{KOH}$ e recém destilada) e cloreto de tosila $(4,0 \mathrm{~g}, 21 \mathrm{mmol})$. A mistura foi mantida sob agitação por $24 \mathrm{~h}$ à temperatura ambiente. Ao final desse tempo, a mistura foi transferida para um béquer $(1000 \mathrm{~mL})$. À mistura, sob agitação magnética, foram adicionadas lentamente pequenas porções de água até obtençãode um precipitado, que foi coletado por filtração a vácuo, lavado com água em abundância ( 1000 mL) e seco em dessecador a vácuo, rendendo um sólido branco $(2,66 \mathrm{~g}, 92 \%)$, p.f. $164-165^{\circ} \mathrm{C}$.

IV $(\mathrm{KBr}) v_{\text {máx }} \mathrm{cm}^{-1}: 2947-2845\left(\mathrm{v}_{\mathrm{CH}^{-}} \mathrm{sp}^{3}\right), 1668\left(\mathrm{v}_{\mathrm{C}=\mathrm{C}}\right), 1754$ ( $v_{\mathrm{CO}}$-éster), 1599-1495 (aromático), 1452-1343 $\left(\delta_{\mathrm{CH} 2}+{ }_{\mathrm{CH}}\right)$, 1335-1171 ( $\left.\mathrm{SO}_{2}\right), 1034\left(v_{\mathrm{C}-\mathrm{O}-\mathrm{C}}\right)$.

$\mathrm{RMN}{ }^{1} \mathrm{H}\left(\mathrm{CDCl}_{3} 200 \mathrm{MHz}\right) \delta:$ 7,87 (d, 2H-tosila-O), 7,37 (d, 2H-tosila-O), 5,33 (m, H-6), 4,94 (m, H-16) 4,34 (m, H-3), 2,47 (s, 3H-tosila-O), 1,30 (d, 3H-21), 0,99 (s, 3H-19) 0,74 (s, 3H-18).

$\mathrm{RMN}{ }^{13} \mathrm{C}\left(\mathrm{CDCl}_{3} 50 \mathrm{MHz}\right) \delta: 181,3(\mathrm{C}-22), 144,4$ (C-1-Ts), 134,4 (C-4-Ts), 138,5 (C-5) 129,7 (CH-3-Ts), 127,4 (CH-2-Ts), 122,7 (CH-6), 82,5 (CH-16), 81,9 (CH-3), 58,7 (CH-17), 54,5 (CH-14), 49,6 (CH-9), 41,3 (C-13), 38,6 ( $\left.\mathrm{CH}_{2}-12\right), 37,8\left(\mathrm{CH}_{2}-4\right)$ 36,7 $\left(\mathrm{CH}_{2}-1\right), 36,2$ (C-10), 35,9 (CH-20), 32,9 $\left(\mathrm{CH}_{2}-15\right), 31,6$ $\left(\mathrm{CH}_{2}-7\right), 30,9(\mathrm{CH}-8), 28,4\left(\mathrm{CH}_{2}-2\right), 21,5\left(\mathrm{CH}_{3}-\mathrm{Ts}\right), 20,1\left(\mathrm{CH}_{2}-\right.$ 11), 19,0 ( $\left.\mathrm{CH}_{3}-19\right), 17,9\left(\mathrm{CH}_{3}-18\right), 13,5\left(\mathrm{CH}_{3}-21\right)$.

\section{3,5-ciclo-6-hidróxi-vespertilina (V2)}

A uma solução de tosilato de vespertilina $(2,5 \mathrm{~g}, 5,01 \mathrm{mmol})$ em acetona $(50 \mathrm{~mL})$ foi adicionada uma solução de acetato de sódio $(2,5 \mathrm{~g}, 24,40 \mathrm{mmol})$ em água $(12,5 \mathrm{~mL})$. A mistura foi deixada em refluxo sob agitação magnética por 6 h. Após resfriamento, evaporou-se aproximadamente metade do solvente e a mistura foi extraída com acetato de etila $(3 \times 30 \mathrm{~mL})$. A fase orgânica foi lavada com água $(2 \times 30 \mathrm{~mL})$, solução saturada de bicarbonato de sódio $(30 \mathrm{~mL})$ e solução saturada de cloreto de sódio $(30 \mathrm{~mL})$. Após secagem com sulfato de sódio e evaporação do solvente a pressão reduzida, foi obtido um sólido branco $(1,71 \mathrm{~g}, 99 \%)$, p.f. $153-156^{\circ} \mathrm{C}$.

IV $(\mathrm{KBr}) v_{\text {máx }} \mathrm{cm}^{-1}: 3530\left(\mathrm{v}_{\mathrm{OH}}\right), 3020\left(\mathrm{v}_{\mathrm{CH}^{-}} \mathrm{sp}^{2}\right)$ 2951-2868 $\left(v_{\mathrm{CH}^{-}}\right.$ $\left.\mathrm{sp}^{3}\right), 1754$ ( $v_{\mathrm{CO}}$-éster $), 1453-1343\left(\delta_{\mathrm{CH} 2}+{ }_{\mathrm{CH} 3}\right), 1049\left(v_{\mathrm{C}-\mathrm{O}-\mathrm{C}}\right)$.

RMN ${ }^{1} \mathrm{H}\left(\mathrm{CDCl}_{3} 200 \mathrm{MHz}\right) \delta:$ 4,98 (m, H-16), 3,28 (m, H6), 1,34 (d, 3H-21), 1,08 (s, 3H-19) 0,82(s, 3H-18), 0,49-0,30 $(\mathrm{m}, 2 \mathrm{H}-4)$.

$\mathrm{RMN}{ }^{13} \mathrm{C}\left(\mathrm{CDCl}_{3} 50 \mathrm{MHz}\right) \delta: 181,3(\mathrm{C}-22) 82,7(\mathrm{CH}-16)$, 73,1 (CH-6), 58,6 (CH-17), 54,8 (CH-14), 47,6 (CH-9), 42,8 (C-10), 41,7 (C-13), 38,6 (C-5), 38,4 ( $\left.\mathrm{CH}_{2}-12\right), 37,0\left(\mathrm{CH}_{2}-7\right)$, 35,9 (CH-20), 33,0 ( $\left.\mathrm{CH}_{2}-1\right), 32,8\left(\mathrm{CH}_{2}-15\right), 29,2(\mathrm{CH}-8), 24,8$ $\left(\mathrm{CH}_{2}-2\right), 24,0(\mathrm{CH}-3), 21,8\left(\mathrm{CH}_{2}-11\right), 19,3\left(\mathrm{CH}_{3}-19\right), 17,9$ $\left(\mathrm{CH}_{3}-18\right), 13,7\left(\mathrm{CH}_{3}-21\right), 11,5\left(\mathrm{CH}_{2}-4\right)$.

\section{3,5-ciclo-6-ceto-vespertilina (V3)}

Em um balão (250 $\mathrm{mL})$ foram adicionados 3,5-ciclo-6hidróxi-vespertilina $(1 \mathrm{~g}, 2,90 \mathrm{mmol})$ e acetona $(80 \mathrm{~mL})$. A solução foi resfriada com uma mistura água/gelo até $15^{\circ} \mathrm{C}$, sob agitação magnética. À mistura reacional foi adicionado o reagente de Jones ( $2 \times 0,66 \mathrm{~mL})$, em intervalo de 5 minutos. Após 5 minutos da segunda adição do oxidante, adicionou-se etanol $(12 \mathrm{~mL})$, permanecendo sob agitação por mais $10 \mathrm{minu}-$ tos. Em seguida, adicionou-se tolueno $(2 \times 130 \mathrm{~mL})$ e a mistura foi mantida sob agitação vigorosa por 30 minutos e filtrada em um funil sinterizado. As porções de tolueno foram reunidas e lavadas com solução saturada de cloreto de sódio $(3 \times 50$ $\mathrm{mL})$, bicarbonato de sódio $5 \%(50 \mathrm{~mL})$ e solução saturada de cloreto de sódio $(50 \mathrm{~mL})$. Após secagem com sulfato de sódio, a solução foi filtrada para um balão $(500 \mathrm{~mL})$, seguida da adição de silicagel 60 (5 g, 0,05-0,20 mm), permanecendo sob 
agitação por $1 \mathrm{~h}$. A mistura foi filtrada e após evaporação do solvente a pressão reduzida, foi obtido um sólido branco (980 $\mathrm{mg}, 98 \%)$, p.f. $242-244^{\circ} \mathrm{C}$.

IV (KBr) $v_{\text {máx }} \mathrm{cm}^{-1}: 3020\left(v_{\mathrm{CH}^{-}} \mathrm{sp}^{2}\right)$ 2949-2868 $\left(v_{\mathrm{CH}^{-}} \mathrm{sp}^{3}\right)$, 1773 ( $v_{\mathrm{CO}}$-éster $), 1679\left(v_{\mathrm{CO}}\right), 1455-1378\left(\delta_{\mathrm{CH} 2}+{ }_{\mathrm{CH} 3}\right)$.

$\mathrm{RMN}{ }^{1} \mathrm{H}\left(\mathrm{CDCl}_{3} 200 \mathrm{MHz}\right) \delta: 5,01(\mathrm{H}-16), 1,33(\mathrm{~d}, 3 \mathrm{H}-21)$ 1,03 (s, 3H-19), 0,81 (s, 3H-18), 0,71 (m, 2H-4).

$\mathrm{RMN}{ }^{13} \mathrm{C}\left(\mathrm{CDCl}_{3} 50 \mathrm{MHz}\right) \delta: 208,6(\mathrm{CO}-6), 180,9$ (C-22), 82,9 (CH-16), 58,6 (CH-17), 54,8 (CH-14), 46,5 (C-10), 46,1 (C-5), 45,9 (CH-9), 44,5 ( $\left.\mathrm{CH}_{2}-7\right), 41,7$ (C-13), 37,9 $\left(\mathrm{CH}_{2}-12\right)$, 35,9 (CH-20), 35,4 (CH-8), 33,9 (CH-3), 33,2 ( $\left.\mathrm{CH}_{2}-1\right), 32,7$ $\left(\mathrm{CH}_{2}-15\right), 25,7\left(\mathrm{CH}_{2}-2\right), 22,0\left(\mathrm{CH}_{2}-11\right), 19,6\left(\mathrm{CH}_{3}-19\right), 17,8$ $\left(\mathrm{CH}_{3}-18\right), 13,7\left(\mathrm{CH}_{3}-21\right), 11,7\left(\mathrm{CH}_{2}-4\right)$.

\section{2,3-en-6-ceto-vespertilina (V4)}

Uma mistura de 3,5-ciclo-6-ceto-vespertilina (744 mg, 2,17 $\mathrm{mmol}$ ), ácido $p$-toluenossulfônico $(99 \mathrm{mg}, 0,57 \mathrm{mmol})$, brometo de sódio (134 mg, 1,30 mmol) em dimetilformamida $(11 \mathrm{~mL}$, recém destilada) foi refluxada por 4 horas. Em seguida, a mistura foi extraída com acetato de etila $(2 \times 30 \mathrm{~mL})$, lavada com água $(3 \times 20 \mathrm{~mL})$ e solução saturada de cloreto de sódio $(30 \mathrm{~mL})$. Após secagem com sulfato de sódio e evaporação do solvente a pressão reduzida, obteve-se um resíduo, que foi solubilizado em diclorometano $(50 \mathrm{~mL})$. À solução foi adicionada silicagel $60(2,5 \mathrm{~g}, 0,05-0,20 \mathrm{~mm})$ permanecendo a mistura sob agitação por $1 \mathrm{~h}$. A mistura foi filtrada em um funil sinterizado e o solvente evaporado a pressão reduzida. O resíduo foi cromatografado em coluna de sílica (10 g), tendo como eluente hexano: acetato de etila 1:1. Após evaporação do solvente a pressão reduzida foi obtido um sólido branco $(605 \mathrm{mg}$, $81 \%)$, p.f. $185-187^{\circ} \mathrm{C}$.

IV $(\mathrm{KBr}) v_{\text {máx }} \mathrm{cm}^{-1}: 3027\left(v_{\mathrm{CH}^{-}} \mathrm{sp}^{2}\right), 2949-2868\left(v_{\mathrm{CH}^{-}} \mathrm{sp}^{3}\right)$, 1770 ( $v_{\mathrm{CO}}$-éster $), 1706\left(v_{\mathrm{CO}}\right), 1455-1342\left(\delta_{\mathrm{CH} 2}+{ }_{\mathrm{CH} 3}\right)$.

$\mathrm{RMN}{ }^{1} \mathrm{H}\left(\mathrm{CDCl}_{3} 200 \mathrm{MHz}\right) \delta: 5,78(\mathrm{~m}, 2 \mathrm{H}-2,3), 5,00(\mathrm{~m}$, H-16), 1,35 (d, 3H-21), 0,82 (s, 3H-18), 0,73 (s, 3H-19).

$\mathrm{RMN}{ }^{13} \mathrm{C}\left(\mathrm{CDCl}_{3} 50 \mathrm{MHz}\right) \delta: 210,7$ (CO-6), 180,9 (C-22), 124,8 (CH-3), 124,2 (CH-2), 82,1 (CH-16), 58,7 (CH-17), 54,6 (CH-14), 53,6 (CH-5), 53,1 (CH-9), 46,9 $\left(\mathrm{CH}_{2}-7\right), 41,8$ (C-13), 39,7 (C-10), 39, $1\left(\mathrm{CH}_{2}-12\right), 37,5\left(\mathrm{CH}_{2}-1\right), 36,7(\mathrm{CH}-8), 35,9$ (CH-20) 32,6 (CH -15$), 21,5\left(\mathrm{CH}_{2}-4\right), 20,3\left(\mathrm{CH}_{2}-11\right), 17,8$ $\left(\mathrm{CH}_{3}-18\right), 13,6\left(\mathrm{CH}_{3}-21\right), 13,4\left(\mathrm{CH}_{3}-19\right)$.

\section{$2 \alpha, 3 \alpha$-di-hidróxi-6-ceto-vespertilina (V5)}

Em um balão (100 mL), foram adicionados 2,3-en-6-cetovespertilina (420 mg, 1,22mmol), N-metilmorfolina-N-óxido (444 mg, 1,22 mmol) e uma mistura de solventes t-butanol/THF/ água 10:8:1 (38 mL). Em seguida, uma solução de $\mathrm{OsO}_{4}(57$ $\mathrm{mg}, 0,22 \mathrm{mmol})$ em THF (1 mL) foi adicionada e a mistura foi mantida sob agitação por $24 \mathrm{~h}$ à temperatura ambiente. À mistura reacional foi adicionada uma solução de $\mathrm{NaHSO}_{3} 32 \%$ (10 $\mathrm{mL}$ ) permanecendo sob agitação por $1 \mathrm{~h}$. Em seguida, a mistura foi extraída com acetato de etila $(2 \times 30 \mathrm{~mL})$ e lavada com solução saturada de cloreto de sódio (3 x $20 \mathrm{~mL})$. Após secagem com sulfato de sódio e evaporação do solvente a pressão reduzida, foi obtido um sólido branco impuro por CCD. Cromatografia em alumina neutra eluída com clorofórmio-acetona 13:1 forneceu o composto desejado. (434mg, $94 \%$ ). p.f. $255^{\circ} \mathrm{C}$.

IV $(\mathrm{KBr}) v_{\text {máx }} \mathrm{cm}^{-1}: 3484\left(\mathrm{v}_{\mathrm{OH}}\right)$ 2952-2872 $\left(\mathrm{v}_{\mathrm{CH}^{-}} \mathrm{sp}^{3}\right), 1748$ $\left(v_{\mathrm{CO}}\right.$-éster $), 1700$ ( $v_{\mathrm{CO}}$-cetona $), 1438-1368\left(\delta_{\mathrm{CH} 2}+{ }_{\mathrm{CH} 3}\right), 1250$ $1041\left(v_{\mathrm{C}-\mathrm{O}-\mathrm{C}}\right)$.

RMN ${ }^{1} \mathrm{H}\left(\mathrm{CDCl}_{3} 90 \mathrm{MHz}\right) \delta:$ 5,01 (m, H-16), 3,98 (m, H-2 $\alpha$ ), 3,70 (m, H-3 $\alpha$ ), 2,68 (m, H-5), 1,34 (d, 3H-21), 0,78 (s, 6H-18,19).

$\mathrm{RMN}{ }^{13} \mathrm{C}\left(\mathrm{CDCl}_{3} 50 \mathrm{MHz}\right) \delta: 211,1(\mathrm{CO}-6), 181,0(\mathrm{C}-22), 82,4$ (CH-16), 68,8 (CH-2), 68,3 (CH-3), 58,7 (CH-17), 54,4 (CH-14), 53,7 (CH-5), 51,3 (CH-9), 46,6 ( $\left.\mathrm{CH}_{2}-7\right), 42,0$ (C-13), 41,0 (CH-8), $37,6\left(\mathrm{CH}_{2}-1\right), 36,7\left(\mathrm{CH}_{2}-12\right), 36,2(\mathrm{CH}-20) 32,8\left(\mathrm{CH}_{2}-15\right), 27,8\left(\mathrm{CH}_{2}-\right.$ 4), 20,7 ( $\left.\mathrm{CH}_{2}-11\right), 17,8\left(\mathrm{CH}_{3}-18\right), 13,6\left(\mathrm{CH}_{3}-21\right), 13,4\left(\mathrm{CH}_{3}-19\right)$.

\section{CONCLUSÃO}

De acordo com a nossa proposta de síntese do análogo de brassinoesteróide a partir de vespertilina, conseguimos obter os intermediários necessários (V1 - V4) em excelentes rendimentos, bem como o composto desejado $2 \alpha, 3 \alpha$-di-hidróxi-6ceto-vespertilina. Além de apresentar as características estruturais dos anéis A e B idênticas a uma série de brassinoesteróides possuidores de atividade brassínica, este novo análogo apresenta ainda uma função oxigenada (lactona) no anel E. Vários ensaios da atividade biológica sugerem que a presença de funções oxigenadas, em C-22 e C-23 da cadeia lateral, assim como ésteres de sistemas pregnanos em C-20 e androstanos em C-17, aumentam a atividade brassínica destes fito-hormônios ${ }^{20}$.

\section{AGRADECIMENTOS}

Os autores agradecem ao Prof. Dr. Edilberto R. Silveira (CENAUREMN-UFC) pelos espectros de ressônancia magnética nuclear de hidrogênio e de carbono, à Universidade Católica de Brasília (UCB), ao Conselho Nacional do Desenvolvimento Científico e Tecnológico (CNPq), ao MCT-RHAE e à Fundação de Apoio à Pesquisa no Distrito Federal (FAPDF) pelo apoio financeiro.

\section{REFERENNCIAS}

1. Mandava, N.; Sidwell, B. A.; Mitchell, J. W.; Worley, J. F.; Ind. Eng. Chem. Prod. Res. Dev. 1973, 12, 138.

2. Gregory, L. E.; Am. J. Bot. 1981, 68, 586.

3. Mitchell, J. W.; Gregory, L. E.; Nature -New Biol. 1972, 239, 253.

4. Grove, M. D.; Spencer, G. F.; Rohwedder, W. K.; Mandava, N.; Worley, J. F.; Warthen, J. D.; Steffens, G. L.; FlippenAnderson, J. L.; Cook, J. C.; Nature 1979, 281, 216.

5. Thompson, M. J.; Mandava, N.; Flippen-Anderson, J. L.; Worley, J. F.; Dutky, S. R.; Robbins, W. E.; Lusby, W.; J. Org. Chem. 1979, 44, 5002.

6. Yokota, T.; Arima, M.; Takahashi, N.; Tetrahedron Lett. 1982, 23, 1275.

7. Ikeda, M., Takatsuto, S., Sassa, T., Ikekawa, N., Nukina, M., Agric. Biol. Chem. 1983, 47, 655.

8. Abe, H., Morishita, M., Uchiama, M., Takatsuto, S., Ikekawa, N., Ikeda, M., Sassa, T., Kitsuwa, T., Marumo, S., Experientia. 1983, 39, 351.

9. Takatsuto, S.; Ying, B.; Morisaki, M.; Ikekawa, N.; J. Cromatogr. 1982, 239, 233.

10. Abe, H.; Morishita, M.; Uchiama, M.; Marumo, S.; Munakata, K.; Takatsuto, S.; Ikekawa, N.; Agric. Biol. Chem. 1982, 46, 2609.

11. Ikeda, M.; Takatsuto, S.; Sassa, T.; Ikekawa, N.; Nukina, M.; Agric. Biol. Chem. 1983, 47, 655.

12. Abe, H.; Morishita, M.; Uchiama, M.; Takatsuto, S.; Ikekawa, N.; Ikeda, M.; Sassa, T.; Kitsuwa, T.; Marumo, S.; Experientia. 1983, 39, 351.

13. Mandava, N.; Ann. Rev. Plant Physiol. Plant Mol. Biol. 1988, 39, 23

14. Yokota, T.; Koba, S. -K.; Takatsuto, S.; Ikekawa, N.; Sakakibara, M.; Okada, K.; Mori, K.; Takahashi, N.; Agric. Biol. Chem. 1987, 51, 1625.

15. Tokota, T.; Takahashi, N.; Plant Growth Substances 1985; Bopp, M., Ed.; Springer-Verlag; Berlin, Heidelberg, New York, 1986; p 129

16. Khripach, V. A.; Pure \& Appl. Chem. 1990, 62, 1319.

17. Takatsuto, S.; Ikekawa, N.; Morishita, T.; Abe, H.; Chem. Pharm. Bull. 1987, 35, 211.

18. Meudt, W. J.; Mandava, N. B.; Plant Physiol. 1982, 69, 12.

19. Adam, G.; Marquardt, V.; Vorbrodt, H. M.; Hörhold, C.; Andreas, W.; Gartz, J. Brassinosteroids: Chemistry, 
bioactivity, and Applications, ACS Symposium series 474, J. Am. Chem. Soc.; Washington, DC, 1991; p 74.

20. ibid, Kohout, L., p 56.

21. Gárate, J. L. M.; Araujo, E. R. de; Magalhães, G. C. de; aceito para publicação na Química Nova

22. Sato, Y.; Latham, H. G.; Mossetig, E.; J. Org. Chem. 1957, 22, 1496.
23. Sato, Y.; Ikekawa, N.; J.Org. Chem. 1961, 26, 5088.

24. Kirk, D. N., Hartshorn, M. P., Steroid Reaction Mechanisms, Elsevier Publishing Company, Amsterdam, monograph 7, 1968; p 228-304

25. Steele, J. A.; Mossetig, E.; J. Org. Chem. 1963, 28, 571.

26. Aburatani, M.; Takeuchi, T.; Mori, K.; Synthesis, 1987, 2,181 . 